\title{
Lymphedema-atrial septal defects-facial changes syndrome
}

INSERM

\section{Source}

INSERM. (1999). Orphanet: an online rare disease and orphan drug data base. Lymphedema-atrial septal defects-facial changes syndrome. ORPHA:86915

Lymphedema-atrial septal defects-facial changes syndrome is characterised by congenital lymphoedema of the lower limbs, atrial septal defect and a characteristic facies (a round face with a prominent forehead, a flat nasal bridge with a broad nasal tip, epicanthal folds, a thin upper lip and a cleft chin). It has been described in two brothers and a sister. Transmission appears to be autosomal recessive. 